\title{
Improvement of postoperative cognitive dysfunction and attention network function of patients with ischemic cerebrovascular disease via dexmedetomidine
}

\author{
${\text { JINGCHAO } \text { ZHANG }^{1 *}, \text { GUOLIANG WANG }}^{2^{*}}$, FANGXIANG ZHANG $^{1}$ and QIAN ZHAO ${ }^{1}$ \\ Departments of ${ }^{1}$ Anesthesiology and ${ }^{2}$ Hepatobiliary Surgery, \\ Guizhou Provincial People's Hospital, Guiyang, Guizhou 550000, P.R. China
}

Received October 10, 2017; Accepted January 4, 2018

DOI: $10.3892 / \mathrm{etm} .2018 .5806$

\begin{abstract}
The protective effect of dexmedetomidine on cognitive dysfunction and decreased attention network function of patients with ischemic cerebrovascular disease after stenting was investigated. Fifty-eight patients with ischemic cerebrovascular disease undergoing stenting in Guizhou Provincial People's Hospital were selected and randomly divided into control group $(n=29)$ and dexmedetomidine group $(n=29)$. The dexmedetomidine group was treated with dexmedetomidine before induced anesthesia, while the control group was given the same dose of normal saline; and the normal volunteers of the same age were selected as the normal group $(n=29)$. At 3 days after operation, the levels of serum S100B and nerve growth factor (NGF) in each group were detected using the enzyme-linked immunosorbent assay, and the level of brainderived neurotrophic factor (BDNF) was detected via western blotting. Montreal cognitive assessment (MoCA) and attention network test (ANT) were performed. Moreover, the cognitive function and attention network function, and the effects of dexmedetomidine on cognitive function and attention network function were evaluated. The concentrations of serum S100B and NGF in dexmedetomidine group was lower than those in control group $(\mathrm{P}<0.01)$. The results of western blotting showed that the levels of serum BDNF in control group and dexmedetomidine group were significantly lower than that in normal group $(\mathrm{P}<0.01)$, and it was higher in dexmedetomidine group than that in control group $(\mathrm{P}<0.01)$. Besides, both MoCA and ANT results revealed that the visual space and executive function scores, attention scores, delayed memory scores, targeted
\end{abstract}

Correspondence to: Dr Jingchao Zhang, Department of Anesthesiology, Guizhou Provincial People's Hospital, 83 Zhongshan Dong Road, Nanming, Guiyang, Guizhou 550000, P.R. China

E-mail: jingchao_1984@126.com

*Contributed equally

Key words: dexmedetomidine, ischemic cerebrovascular disease, cognitive dysfunction, attention network function network efficiency and executive control network efficiency in dexmedetomidine group were obviously higher than those in control group $(\mathrm{P}<0.01)$. The cognitive function and attention network function of patients with ischemic cerebrovascular disease have a certain degree of damage, and the preoperative administration of dexmedetomidine can effectively improve the patient's cognitive dysfunction and attention network function after operation.

\section{Introduction}

Ischemic cerebrovascular disease can lead to hemiplegia or even death. According to epidemiological data, ischemic cerebrovascular disease ranks third in the diseases causing the death of the elderly, seriously affecting the life quality and life health of patients $(1,2)$. Clinically, the ischemic cerebrovascular disease is often treated with surgery supplemented with drug therapy, and a large number of studies have shown that patients with ischemic cerebrovascular disease will suffer from vascular cognitive dysfunction; reducing the thrombosis via early diagnosis can effectively prevent the occurrence of vascular cognitive dysfunction (3-5). Stenting can be effective in the treatment of ischemic cerebrovascular disease, which is widely used in clinic due to its definite efficacy and small trauma (6). Stenting for patients with ischemic cerebrovascular disease may lower the risk of vascular cognitive dysfunction of patients. However, El Hammi et al (7) found that the cognitive function of patients with ischemic cerebrovascular disease treated with stenting still has a certain degree of cognitive dysfunction and damage to attention network function compared with normal people. Li and Liu (8) found through detecting the levels of serum S100B, nerve growth factor (NGF) and brain-derived neotrophic factor (BDNF) that dexmedetomidine provides significant improvement on the recovery of cognitive function of patients receiving epilepsy foci resection, and S100B, NGF and BDNF are highly sensitive and can accurately predict the patient's brain damage. The effects of dexmedetomidine on recovery of cognitive function and attention network function of patients with ischemic cerebrovascular disease have not been studied. Therefore, in this study, the cognitive function and attention network function of patients with ischemic cerebrovascular disease after operation 
were studied, expecting to clarify the effects of dexmedetomidine on the cognitive dysfunction and attention network function of patients with ischemic cerebrovascular disease, and to provide a theoretical basis and related guidance for the recovery of patients with ischemic cerebrovascular disease.

\section{Patients and methods}

Patients of the study. The samples in this study were from patients diagnosed as ischemic cerebrovascular disease by deputy director and above in Guizhou Provincial People's Hospital from March 2015 to September 2016. Inclusion criteria: 1) patients diagnosed as ischemic cerebrovascular disease via head CT or MRI; 2) patients without obvious hemiplegia, with normal language skills; 3 ) patients who could complete the cognitive and attention network function tests; 4) patients who agreed to receive stenting, signed the informed consent and were willing to participate in this experimental study. Fifty-eight patients meeting inclusion criteria were selected, and they were aged 48-73 years, including 31 males and 27 females. Other wasting diseases were excluded from patients enrolled, and the clinical and pathological data and complete treatment program of the above patients in the treatment process were retained. The study was approved by the Ethics Committee of Guizhou Provincial People's Hospital.

Experimental grouping. The above patients selected were randomly divided into control group $(n=29)$ and dexmedetomidine group ( $\mathrm{n}=29)$. The dexmedetomidine group was treated with intravenous administration of $1 \mu \mathrm{g} / \mathrm{kg}$ dexmedetomidine using a micro-injection pump before induced anesthesia, while the control group was given the same dose of normal saline. Other operative procedures and treatment regimens were the same, and the normal volunteers at the same age were selected in the same period as the normal group; the cognitive function and attention network function of patients were analyzed.

Detection of serum S100B and NGF levels via enzyme-linked immunosorbent assay (ELISA). The peripheral venous blood was drawn at 3, 7 and 30 days after operation, and $2 \mathrm{ml}$ blood was collected after separation of serum to detect the levels of serum S100B and NGF strictly according to the S100B and NGF ELISA kit (Boster Biological Technology Co. Ltd., Wuhan, China). The remaining serum was stored at $-80^{\circ} \mathrm{C}$ for subsequent experiments.

Serum BDNF level. Serum specimens stored at $-80^{\circ} \mathrm{C}$ and collected from each group of patients were thawed and the total protein was extracted after addition of RIPA lysate (1:1; Beyotime Biotechnology Co., Ltd., Shanghai, China), the protein content of each group was detected using the BCA protein quantification kit (Thermo Fisher Scientific, Waltham, MA, USA), and the same concentration of loading sample was prepared, followed by preparation of $10 \%$ sodium dodecyl sulfate-polyacrylamide separation gel and spacer gel and electrophoresis separation. Then the protein was transferred onto the PVD membrane and sealed in 5\% skimmed milk powder for $1 \mathrm{~h}$. The target bands were cut and incubated with BDNF and GAPDH antibodies (diluted at 1:1,000; cat. nos. SAB1405514 and SAB1405848; Sigma) at $4^{\circ} \mathrm{C}$ overnight. The bands were
Table I. General data analysis

\begin{tabular}{lccc}
\hline Characteristics & $\begin{array}{c}\text { Control } \\
\text { group }\end{array}$ & $\begin{array}{c}\text { Dexmedetomidine } \\
\text { group }\end{array}$ & P-value \\
\hline Age (years) & $56.27 \pm 8.32$ & $59.76 \pm 7.57$ & $>0.05$ \\
Sex (male/female) & $15 / 14$ & $16 / 13$ & $>0.05$ \\
Hypertension (\%) & 68.96 & 72.41 & $>0.05$ \\
Diabetes mellitus (\%) & 34.48 & 31.03 & $>0.05$ \\
Drinking (\%) & 41.37 & 44.82 & $>0.05$ \\
\hline
\end{tabular}

washed with Tris-buffered saline Tween (TBST) 3 times (10 min each time), and the rabbit anti-mouse secondary polyclonal antibody (diluted at 1:5,000; cat. no. SAB3701038; Sigma) were incubated at room temperature for $2 \mathrm{~h}$. After that, bands were washed with TBST again 3 times (10 min each time). The DAB coloring solution was added in the dark room for development and fixation, and the gray value of band was analyzed using the gel imaging system. The BDNF/GAPDH ratio indicated the expression level of BDNF protein in serum.

Montreal cognitive assessment (MoCA). The cognitive function was evaluated via MoCA in an independent and quiet environment, including visual space and executive function: 1 point, the patients can describe the profile of clock, record the number order and position, and identify the distinction between the hour hand and the minute hand; 0 point, the patients cannot identify as above. Attention score: 1 point, the patient can repeat a series of figures said by the experimental staff; 0 point, the patient cannot repeat; 1 point, the patients can quickly find the difference of figures between the first and second time; 0 point, the patients cannot recognize that. Continuous subtraction (100 minus 6) is performed for a total of 5 times; 3 points, one error or normal; 2 points, 2 or 3 errors; 1 point, 4 errors; 0 point, all errors. Delayed memory score: 1 point for each of 5 words recalled by the patients.

Attention network test (ANT). The attention network function was evaluated via ANT in an independent and quiet environment, including the targeted network efficiency and executive control network efficiency. The patients were trained and tested in strict accordance with the training and testing process of ANT. The patients were stimulated using the E-Prime 2.0 program; the thumb was placed on the number key 1 and 3 to determine the direction of target arrow. The patient's attention network function was evaluated based on the targeted network efficiency and executive control network efficiency of patients.

Statistical analysis. The data in this study are presented as mean \pm standard deviation, and SPSS 19.0 software (SPSS Inc., Chicago, IL, USA) was used for data processing. The t-test was used for intergroup comparison, while the analysis of variance was used for comparison among groups. The homogeneity test of variance was performed; Bonferroni method was used for pairwise comparison if the variance was homogeneous; otherwise, Welch method was used for analysis. Dunnett's T3 method was used for multiple comparisons. $\mathrm{P}<0.05$ was considered to indicate a statistically significant difference. 


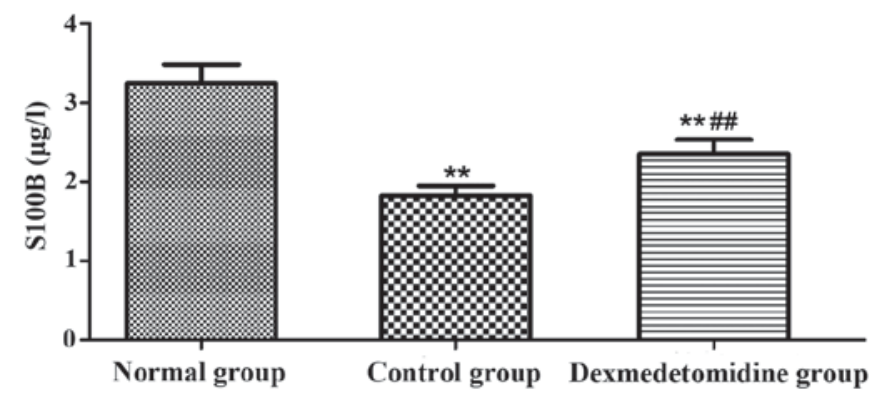

Figure 1. Content of S100B; the contents of serum S100B in control group and dexmedetomidine group are significantly lower than that in normal group $\left({ }^{* *} \mathrm{P}<0.01\right)$, and it is significantly higher in dexmedetomidine group than that in control group $\left({ }^{\# \#} \mathrm{P}<0.01\right)$.

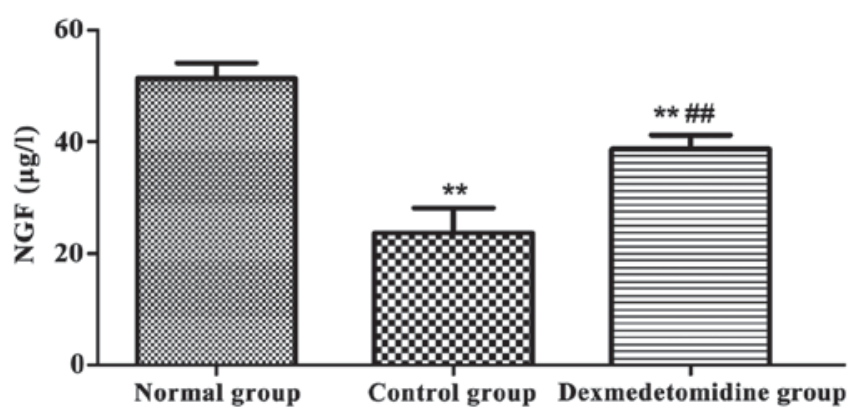

Figure 2. Content of NGF; the contents of serum NGF in control group and dexmedetomidine group are significantly lower than that in normal group $\left({ }^{* *} \mathrm{P}<0.01\right)$, and it is significantly higher in dexmedetomidine group than that in control group $\left({ }^{\# \#} \mathrm{P}<0.01\right)$.

\section{Results}

General data analysis. The general data of patients in control group and dexmedetomidine group are recorded in detail and analyzed (Table I). The age, sex, percentage of hypertension history, percentage of diabetes mellitus history, percentage of drinking history and percentage of smoking history had no statistically significant differences between control group and dexmedetomidine group $(\mathrm{P}>0.05)$.

Serum S100B and NGF levels. The levels of serum S100B and NGF in each group were detected using the ELISA kit (Figs. 1 and 2). Compared with those in normal group, the levels of serum S100B and NGF in control group and dexmedetomidine group were significantly decreased $(\mathrm{P}<0.01)$, and they were significantly higher in dexmedetomidine group than those in control group $(\mathrm{P}<0.01)$.

Serum BDNF level. The level of serum BDNF in each group was detected via western blotting (Fig. 3). The levels of serum BDNF in control group and dexmedetomidine group were significantly decreased compared with that in normal group $(\mathrm{P}<0.01)$, and it was higher in dexmedetomidine group than that in control group $(\mathrm{P}<0.01)$.

MoCA scores of patients in each group. MoCA was used to record the visual space and executive function scores, attention score and delayed memory score of each group in detail in
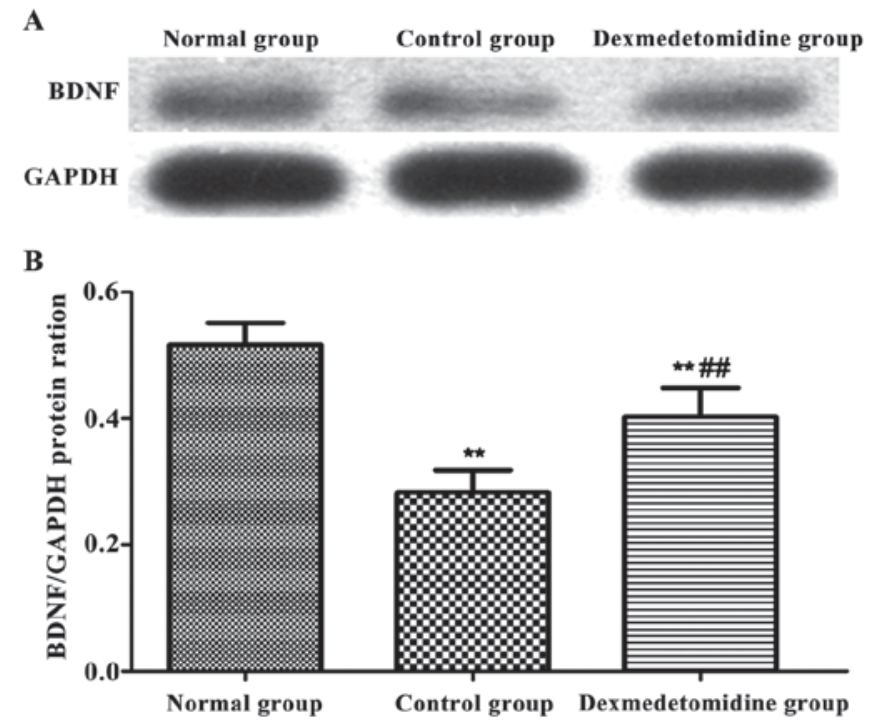

Figure 3. Detection of serum BDNF protein level via western blotting (A) Protein band chart; (B) statistical chart; the levels of serum BDNF protein in control group and dexmedetomidine group are significantly lower than that in normal group $\left({ }^{* *} \mathrm{P}<0.01\right)$, and it is higher in dexmedetomidine group than that in control group $\left({ }^{\# \#} \mathrm{P}<0.01\right)$.

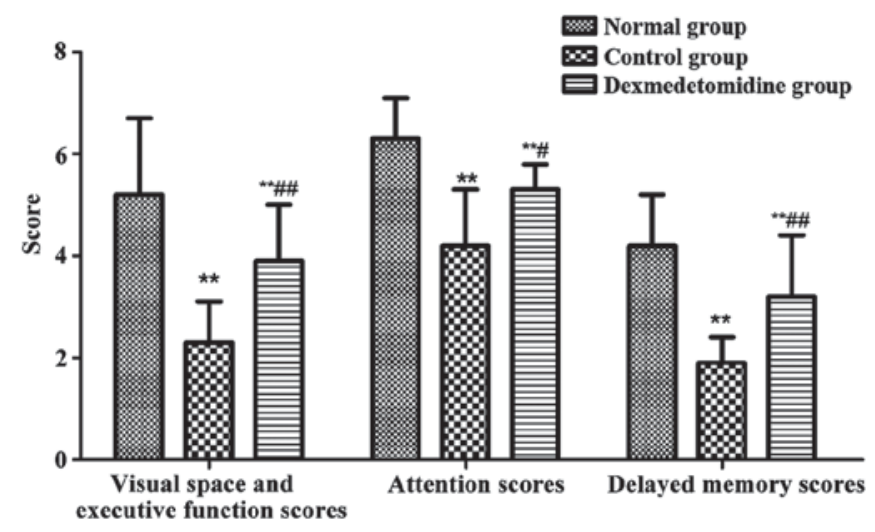

Figure 4. MoCA score; the visual space and executive function scores, attention scores and delayed memory scores in control group and dexmedetomidine group are obviously lower than those in normal group $\left({ }^{* *} \mathrm{P}<0.01\right)$, and they are obviously higher in dexmedetomidine group than those in control group $\left({ }^{\#} \mathrm{P}<0.05,{ }^{\# /} \mathrm{P}<0.01\right)$.

the experiment, and the cognitive function of patients was evaluated (Fig. 4). The visual space and executive function scores, attention scores and delayed memory scores in control group and dexmedetomidine group were obviously decreased compared with those in normal group $(\mathrm{P}<0.01)$, and they were obviously higher in dexmedetomidine group than those in control group $(\mathrm{P}<0.01, \mathrm{P}<0.05)$.

ANT of patients in each group. ANT was used to record the targeted network efficiency and control network efficiency of each group (Fig. 5). The targeted network efficiency and control network efficiency in control group and dexmedetomidine group were obviously decreased compared with those in normal group $(\mathrm{P}<0.01)$, and they were obviously higher in dexmedetomidine group than those in control group $(\mathrm{P}<0.01$, $\mathrm{P}<0.05)$. 


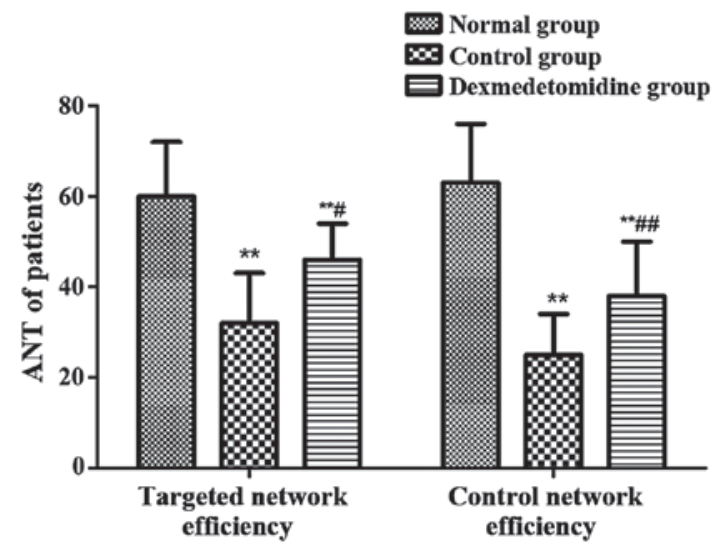

Figure 5. Attention network test (ANT); the targeted network efficiency and control network efficiency in control group and dexmedetomidine group are obviously lower than those in normal group $\left({ }^{* *} \mathrm{P}<0.01\right)$, and they are obviously higher in dexmedetomidine group than those in control group $\left({ }^{\#} \mathrm{P}<0.05\right.$, $\left.{ }^{\# \#} \mathrm{P}<0.01\right)$.

\section{Discussion}

Patients with ischemic cerebrovascular disease will suffer from dysfunction or deletions in memory, attention, perception and feeling, making it impossible for patients to participate in the normal social activities and daily work and life, seriously affecting the life quality of patients; the above symptoms are collectively referred to as cognitive dysfunction (9). Researchers proposed the attention network theory by analyzing a large number of research results of cognitive psychology and brain function imaging, and evaluated the network function with the targeted network efficiency and control network efficiency $(10,11)$. Cognitive function evaluation and attention network function evaluation are applied in the research on patients with stroke, Parkinson's disease, epilepsy and Alzheimer's disease (12-14). The mechanism of cognitive dysfunction and attention network damage in patients with cerebrovascular disease is not clear, and some studies have reported that the possible mechanism may be the vascular inflammation, cerebrovascular injury, environmental factors and genetic factors $(15,16)$. In recent years, clinicians and researchers have paid increased attention to the cognitive dysfunction and attention network deletion in patients with central nervous system diseases, which is helpful to recover the patients' cognitive function and attention network function.

In this study, the cognitive function and attention network function of patients with ischemic cerebrovascular disease after stenting were evaluated using the classical MoCA and ANT. The results showed that patients with ischemic cerebrovascular disease will suffer from severe cognitive dysfunction and attention network damage after operation. Yang and Rosenberg (17) found that the cognitive dysfunction and attention network function damage will be recovered in patients with carotid atherosclerosis after stenting, but it was found in this study that the cognitive function and attention network function of patients with ischemic cerebrovascular disease still have obvious abnormality compared with normal people even after stenting.

In this study, patients with ischemic cerebrovascular disease received intravenous administration of dexmedetomidine before treatment, and the surgical process and treatment protocol were the same as those of the control group. After operation, the serum S100B, NGF and BDNF expression levels in patients were detected, and the cognitive function and attention network function were evaluated. The results showed that the levels of serum S100B, NGF and BDNF in dexmedetomidine group were significantly higher than those in control group, and the cognitive function and attention network function were also significantly superior to those in control group. The above results indicated that dexmedetomidine has a significant protective effect on patients with ischemic cerebrovascular disease and contributes to the recovery of cognitive function and attention network function of patients with of ischemic cerebrovascular disease. Moreover, the cognitive function and attention network function are closely related to the brain tissue structure and brain blood flow distribution; if the brain tissues are in an ischemia and hypoxia state for a long time, there will be severe cognitive dysfunction and attention network function damage $(18,19)$.

Dexmedetomidine is a highly-selective excitatory center and peripheral $\alpha 2$ receptor drug, which can make the blood pressure change stable under the stimulation and the blood flow more stable. Substantial research evidence reveals that dexmedetomidine has a significant protective effect on brain, which can significantly reduce the coma time and promote the postoperative cognitive function recovery of patients with brain injury (20). Dexmedetomidine can contract the vessels and produce sedative and analgesic effects. The latest research data show that dexmedetomidine can affect the expression levels of inflammatory factors and reduce the inflammatory response in blood vessels through activating the corresponding signal pathway (21). In this study, the protective effect of dexmedetomidine on cerebral blood vessels may be also manifested as reducing the level of inflammatory factors in cerebral blood vessels, reducing the level of inflammation in brain tissues of patients with ischemic cerebrovascular disease and reducing the damage of inflammation to the brain tissues, thus improving the cognitive function and attention network function.

In conclusion, patients with ischemic cerebrovascular disease will suffer from obvious cognitive dysfunction and attention network function damage after stenting, and the preoperative administration of dexmedetomidine can effectively protect the cerebral blood vessels, improve the cognitive dysfunction and attention network function damage, and increase the postoperative life quality of patients.

\section{Acknowledgements}

Not applicable.

Funding

No funding was received.

\section{Availability of data and materials}

The datasets used and/or analyzed during the present study are available from the corresponding author on reasonable request. 


\section{Authors' contributions}

JZ was mainly responsible for the study design and manuscript writing. QZ contributed to the conception and design of the study. GW collected and assembled the data. FZ mainly participated in the collection of the data and the follow-up management of the patients. QZ reviewed and finalized the manuscript. All authors read and approved the final manuscript.

\section{Ethics approval and consent to participate}

The study was approved by the Ethics Committee of Guizhou Provincial People's Hospital. Signed written informed consents were obtained from the patients.

\section{Consent for publication}

Not applicable.

\section{Competing interests}

The authors declare that they have no competing interests.

\section{References}

1. Wong TS, Liao KF, Lin CM, Lin CL, Chen WC and Lai SW: Chronic pancreatitis correlates with increased risk of cerebrovascular disease: A retrospective population-based cohort study in Taiwan. Medicine (Baltimore) 95: e3266, 2016.

2. Huang R, Hu Z, Feng Y, Yu L and Li X: The transcription factor IRF6 co-represses PPAR $\gamma$-mediated cytoprotection in ischemic cerebrovascular endothelial cells. Sci Rep 7: 2150, 2017.

3. Di Napoli M and McLaughlin B: The ubiquitin-proteasome system as a drug target in cerebrovascular disease: Therapeutic potential of proteasome inhibitors. Curr Opin Investig Drugs 6: 686-699, 2005.

4. Fancellu L, Borsini W, Romani I, Pirisi A, Deiana GA, Sechi E, Doneddu PE, Rassu AL, Demurtas R, Scarabotto A, et al: Exploratory screening for Fabry's disease in young adults with cerebrovascular disorders in northern Sardinia. BMC Neurol 15: 256, 2015.

5. Xu X, Ma H, Xu J, Huang $\mathrm{H}$, Wu X, Xiong $\mathrm{Y}$, Zhan $\mathrm{H}$ and Huang F: Elevation in circulating YKL-40 concentration in patients with cerebrovascular disease. Bosn J Basic Med Sci 14: 120-124, 2014.

6. Russo C, Jin Z, Liu R, Iwata S, Tugcu A, Yoshita M, Homma S, Elkind MS, Rundek T, Decarli C, et al: LA volumes and reservoir function are associated with subclinical cerebrovascular disease: The CABL (Cardiovascular Abnormalities and Brain Lesions) study. JACC Cardiovasc Imaging 6: 313-323, 2013.

7. El Hammi E, Samp J, Rémuzat C, Auray JP, Lamure M, Aballéa S, Kooli A, Akhras K and Toumi M: Difference of perceptions and evaluation of cognitive dysfunction in major depressive disorder patients across psychiatrists internationally. Ther Adv Psychopharmacol 4: 22-29, 2014
8. Li Y and Liu S: The effect of dexmedetomidine on oxidative stress response following cerebral ischemia-reperfusion in rats and the expression of intracellular adhesion molecule-1 (ICAM-1) and S100B. Med Sci Monit 23: 867-873, 2017.

9. Santos CY, Snyder PJ, Wu WC, Zhang M, Echeverria A and Alber J: Pathophysiologic relationship between Alzheimer's disease, cerebrovascular disease, and cardiovascular risk: A review and synthesis. Alzheimers Dement Amst 7: 69-87, 2017.

10. Francoeur RB: Symptom profiles of subsyndromal depression in disease clusters of diabetes, excess weight, and progressive cerebrovascular conditions: A promising new type of finding from a reliable innovation to estimate exhaustively specified multiple indicators-multiple causes (MIMIC) models. Diabetes Metab Syndr Obes 9: 391-416, 2016.

11. Lee K, Kim H, Heo JH, Bae HJ, Koh IS and Chang S: Application of magnetic resonance imaging and magnetic resonance angiography as diagnostic measures for the first attack of suspected cerebrovascular diseases in Korea. Yonsei Med J 52: 727-733, 2011.

12. Perneczky R, Tene O, Attems J, Giannakopoulos P, Ikram MA, Federico A, Sarazin M and Middleton LT: Is the time ripe for new diagnostic criteria of cognitive impairment due to cerebrovascular disease? Consensus report of the International Congress on Vascular Dementia working group. BMC Med 14: 162, 2016.

13. Manukhina EB, Downey HF, Shi X and Mallet RT: Intermittent hypoxia training protects cerebrovascular function in Alzheimer's disease. Exp Biol Med (Maywood) 241: 1351-1363, 2016.

14. Daulatzai MA: Pathogenesis of cognitive dysfunction in patients with obstructive sleep apnea: A hypothesis with emphasis on the nucleus tractus solitarius. Sleep Disord 2012: 251096, 2012.

15. Behrouz R, Malek AR and Torbey MT: Small vessel cerebrovascular disease: The past, present, and future. Stroke Res Treat 2012: 839151, 2012.

16. Humpel C: Chronic mild cerebrovascular dysfunction as a cause for Alzheimer's disease? Exp Gerontol 46: 225-232, 2011.

17. Yang Y and Rosenberg GA: Blood-brain barrier breakdown in acute and chronic cerebrovascular disease. Stroke 42: 3323-3328, 2011.

18. Takahashi H, Xia P, Cui J, Talantova M, Bodhinathan K, Li W, Saleem S, Holland EA, Tong G, Piña-Crespo J, et al: Pharmacologically targeted NMDA receptor antagonism by NitroMemantine for cerebrovascular disease. Sci Rep 5: 14781 , 2015.

19. Pannarale G, Moroni C, Acconcia MC, Pannitteri G, Truscelli G, Valente L, Gentile P, Lopreiato F, Licitra R, Tancredi M, et al: The natural history of prehypertension. A 20-year follow-up. Eur Rev Med Pharmacol Sci 21: 1329-1334, 2017.

20. Choi IY, Hwang L, Jin JJ, Ko IG, Kim SE, Shin MS, Shin KM, Kim CJ, Park SW, Han JH, et al: Dexmedetomidine alleviates cerebral ischemia-induced short-term memory impairment by inhibiting the expression of apoptosis-related molecules in the hippocampus of gerbils. Exp Ther Med 13: 107-116, 2017.

21. Zeng X, Wang H, Xing X, Wang Q and Li W: Dexmedetomidine protects against transient global cerebral ischemia/reperfusion induced oxidative stress and inflammation in diabetic rats. PLoS One 11: e0151620, 2016.

This work is licensed under a Creative Common Attribution-NonCommercial-NoDerivatives 4.0 International (CC BY-NC-ND 4.0) License. 